\title{
Tuberculosis trends over a five-year period at a tertiary care university-affiliated hospital in Singapore
}

Suzanna Binte $\underline{\text { Jappar }}^{1}$, BN, Su Ying $\underline{\text { Low }}^{1}$, BMBCh, MRCP

INTRODUCTION Tuberculosis (TB), a potentially fatal infectious disease, poses significant health problems. The objective of this study was to analyse trends among newly diagnosed TB patients seen at the Singapore General Hospital (SGH). METHODS This study was a retrospective case record review of notified TB patients at SGH between 2006 and 2010 (inclusive).

RESULTS A total of 1,979 cases of TB were notified over the five years. The number increased from 368 in 2006 to 407 in 2010 ( $p=0.51$ ), in tandem with the increasing number of patients seen at the hospital and the rising population in Singapore. Singaporean residents accounted for $82.8 \%$ of the cases, while non-Singaporean residents and non-residents made up the remainder. The year-to-year percentage of non-Singaporean residents and non-residents notified remained stable throughout the five years $(p=0.783)$. The number of male Singaporean residents $(70.7 \%)$ notified was more than twice that of female Singaporean residents (29.3\%). The majority of patients infected with TB (31.9\%) were elderly patients aged $>65$ years. Although testing for the human immunodeficiency virus increased from $43.8 \%$ (2006) to 64.2\% (2010), the number detected with coinfection remained stable (14 and 12 patients in 2006 and 2010, respectively). Mortality rates also remained stable at $12.0 \%$.

CONCLUSION We cannot afford complacency, as there was no downward trend in the number of notified TB cases at SGH from 2006 to 2010. As the elderly contribute significantly to the TB burden, increased efforts are required to manage this vulnerable population.

Keywords: elderly, HIV, trends, tuberculosis

\section{INTRODUCTION}

Tuberculosis (TB) is a potentially fatal infectious disease caused by the Mycobacterium tuberculosis bacterium. Despite the availability of effective drug treatments, TB remains an international health problem and is the second leading cause of death from an infectious disease after human immunodeficiency virus (HIV) infection. ${ }^{(1)}$ In 2012, an estimated 8.6 million people developed TB and 1.3 million died from the disease; Southeast Asian countries accounted for $29 \%$ of the TB cases worldwide. ${ }^{(1)}$ Although Singapore has the lowest incidence of TB (41.5 cases per 100,000 population of residents and long-stayers) among the Southeast Asian countries, ${ }^{(2)}$ TB control continues to remain a challenge. ${ }^{(3)}$ For this reason, our study aimed to analyse trends among newly diagnosed TB patients who were seen at the Singapore General Hospital (SGH).

\section{METHODS}

The case records of all patients with TB notified at SGH from 2006 to 2010 were retrospectively reviewed. TB incidence and patient demographics were compared on a yearly basis and analysed using IBM SPSS Statistics version 21.0 (IBM Corp, Armonk, NY, USA). Study approval was obtained from the SingHealth Centralised Institutional Review Board C.

\section{RESULTS}

A total of 1,979 cases of TB were notified at SGH over the five years. In tandem with the increasing number of patients seen at $\mathrm{SGH}$ and the rising population in Singapore, the number of patients notified to have TB increased from 368 cases in 2006 to 407 cases in 2010 (Table I); this increase was not statistically significant $(p=0.51)$. Singaporean residents accounted for $1,638(82.8 \%)$ of the cases, while non-Singaporean residents and non-residents made up the remaining 341 (17.2\%) cases. The percentage of non-Singaporean residents and nonresidents notified to have TB remained stable over the five years $(p=0.783)$

Among the Singaporean residents notified to have TB at SGH, $77.1 \%$ were Chinese, $14.6 \%$ were Malay, $6.0 \%$ were Indian and $2.3 \%$ were of other races. Male Singaporean residents $(70.7 \%)$ made up more than twice the number of cases as compared to female Singaporean residents $(29.3 \%)$. In contrast, the gender distribution was almost equal $(46.0 \%$ men; $54.0 \%$ women) among the non-Singaporean residents and non-residents who were notified to have TB at SGH (Fig. 1). When the 1,979 cases were categorised according to age, we found that most of the cases involved elderly patients aged $>65$ years (31.9\%) (Fig. 2). Non-Singaporeans and non-residents comprised $51.2 \%$ of the cases within the 20-29 years age category (Fig. 3).

Extrapulmonary TB was diagnosed in 537 of the 1,979 cases (27.1\%), at the sites shown in Table II. Among the 537 patients with extrapulmonary TB, 53 (9.9\%) were immunocompromised - 25 (47.2\%) were HIV positive, 15 (28.3\%) were on immunosuppressive agents (eight post transplantation and seven for rheumatological conditions), 10 (18.9\%) had haematological

${ }_{1}^{1}$ Department of Respiratory and Critical Care Medicine, Singapore General Hospital, Singapore

Correspondence: Ms Suzanna Binte Jappar, TB Programme Coordinator, Department of Respiratory and Critical Care Medicine, Singapore General Hospital, 20 College Road, The Academia, Singapore 169856. suzanna.jappar@sgh.com.sg 
Table I. Total number of patients seen and cases of TB notified at SGH, and Singapore's population between 2006 and 2010.

\begin{tabular}{|c|c|c|c|c|c|}
\hline Parameter & 2006 & 2007 & 2008 & 2009 & 2010 \\
\hline Total no. of patients seen at SGH & 712,779 & 726,897 & 746,843 & 747,597 & 762,417 \\
\hline \multicolumn{6}{|l|}{ No. of TB cases notified at SGH } \\
\hline Total & 368 & 351 & 440 & 413 & 407 \\
\hline Singaporean residents & 305 & 289 & 365 & 349 & 330 \\
\hline Non-Singaporean residents and non-residents & 63 & 62 & 75 & 64 & 77 \\
\hline
\end{tabular}

*Data obtained from the Singapore Department of Statistics. ${ }^{(4)}$ SGH: Singapore General Hospital; TB: tuberculosis

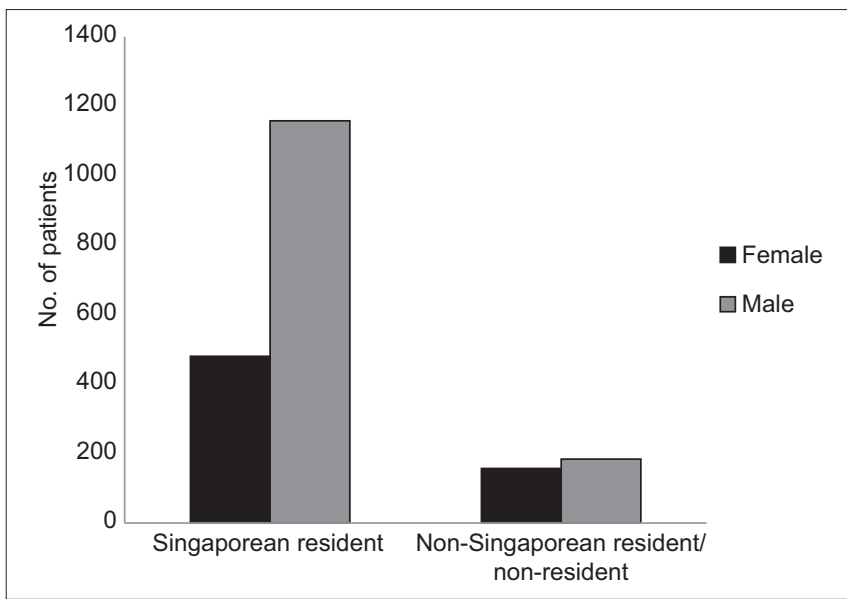

Fig. 1 Bar graph shows the distribution of patients with tuberculosis according to their gender and residency status $(n=1,979)$.

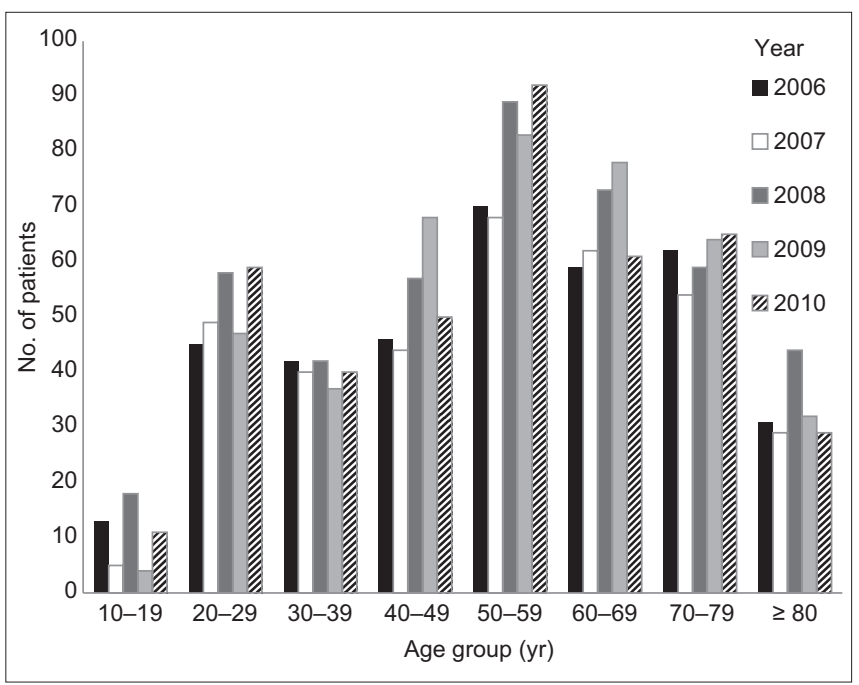

Fig. 2 Bar graph shows the distribution of patients with tuberculosis (TB) according to their age groups and year of TB notification.

malignancies and 3 (5.7\%) were undergoing chemotherapy for cancer. Analysis of the variables did not show any year-to-year change in terms of racial distribution, gender, age and percentage of extrapulmonary TB cases.

HIV testing of patients who were newly diagnosed with TB showed an upward trend from $43.8 \%$ in 2006 to $64.2 \%$ in 2010 (Fig. 4). The actual number of patients detected with coinfection remained stable - 14 in 2006 and 12 in 2010 . Among the patients tested for HIV, $8.7 \%$ and $4.7 \%$ were found to be positive in 2006 and 2010, respectively. Based on the assumption that patients
Table II. Sites of extrapulmonary tuberculosis $(n=537)$.

\begin{tabular}{lc}
\hline Site & No. (\%) \\
\hline Lymph node & $189(35.2)$ \\
Pleura & $157(29.2)$ \\
Musculoskeletal & $63(11.7)$ \\
Gastrointestinal & $60(11.2)$ \\
Genitourinary & $44(8.2)$ \\
Disseminated & $12(2.2)$ \\
Central nervous system & $7(1.3)$ \\
Others & $5(0.9)$ \\
\hline
\end{tabular}

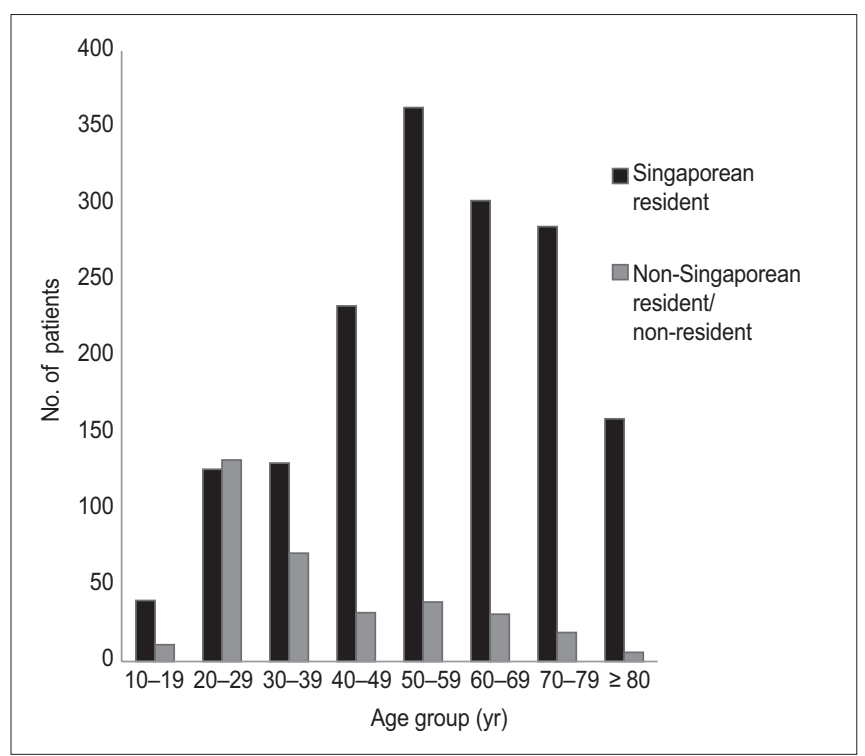

Fig. 3 Bar graph shows the distribution of patients with tuberculosis according to their age and residency status.

who did not test for HIV were negative for HIV, the coinfection rate of HIV with TB would be 3.8\% (14/368) in 2006 and 2.9\% (12/407) in 2010. HIV testing was performed for $58.1 \%$ of the patients with extrapulmonary TB and among those who were tested, $8.0 \%$ were positive for HIV. Assuming that the patients who did not test for HIV were HIV negative, the HIV coinfection rate with extrapulmonary TB would be $4.7 \%$.

Among the 1,979 cases of TB notified, $44.5 \%$ were treated at $\mathrm{SGH}$, while the care of $29.8 \%$ was transferred to other health institutions and $8.9 \%$ left the country. The diagnosis of TB was revised for $89(4.5 \%)$ patients - 86 patients were found to have nontuberculous mycobacteria, one patient had granulomatous inflammation from a lymph node biopsy but cultures for TB and clinical examination were negative, and the last two patients who 


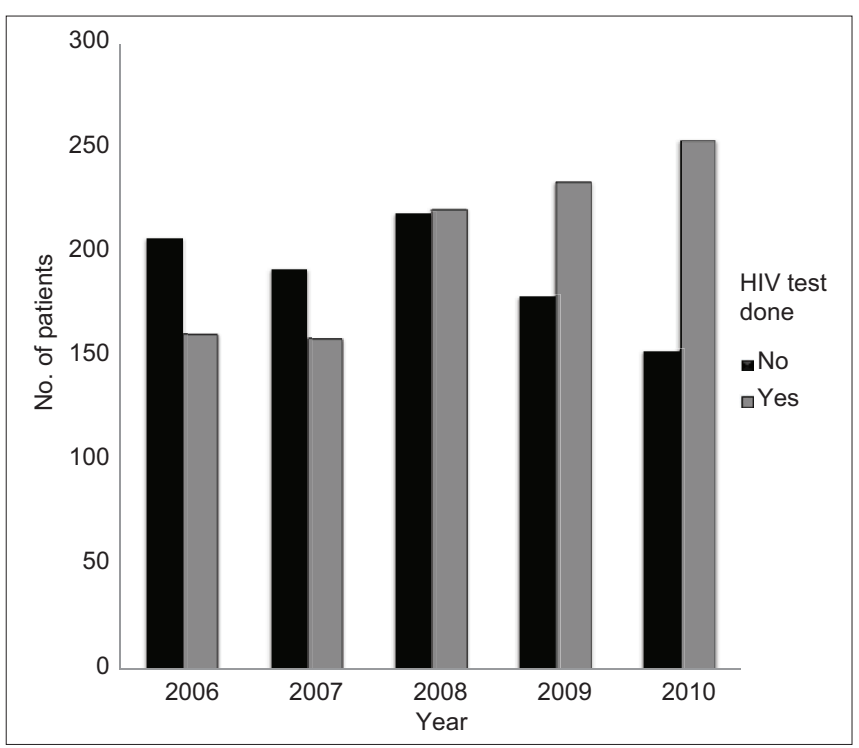

Fig. 4 Bar graph shows the distribution of patients who had HIV tests done in the years 2006-2010.

were initially clinically diagnosed with TB did not show temporal response to treatment.

The overall mortality rate was $12.0 \%$ and remained stable throughout the five years; $28(1.4 \%)$ patients died from TB, while the remaining $210(10.6 \%)$ patients died from other causes, mainly cancer, pneumonia, ischaemic heart disease and sepsis. Among the patients who died from TB, the mean age was $59.7 \pm 22.5$ years, $67.9 \%$ were male, $75.0 \%$ were smear positive for acid fast bacilli and $17.9 \%$ were immunocompromised (two patients were HIV positive, two were on immunosuppressive agents for rheumatological conditions and one was on chemotherapy for cancer). Among these 28 patients, 16 had fulminant pulmonary TB, eight had disseminated TB, two had massive haemoptysis and two had TB meningitis. 22 out of the 28 patients $(78.6 \%)$ were diagnosed at their first presentation to the hospital, while the remaining 6 (21.4\%) patients were diagnosed at a median of 1.5 (range 1-6) months after presentation to the hospital (the delay in diagnosis was due to confounding medical illnesses). Of the 28 patients, 10 (35.7\%) died in the intensive care unit.

\section{DISCUSSION}

SGH is a tertiary care referral centre that services specific geographic parts of Singapore. It also has busy haematology, oncology, rheumatology and transplant units that care for a large population of immunocompromised patients. Although SGH accounts for approximately $20 \%$ of the TB notifications in Singapore, ${ }^{(2)}$ the patient profiles obtained in the present study may not be reflective of TB patients in Singapore as a whole.

Despite the efforts made to control TB, we did not observe a downward trend in the number of TB patients notified at SGH from 2006 to 2010. Similarly, from 2011 to 2013, the number of TB patients notified at SGH did not change significantly 383, 436 and 373 cases in 2011, 2012 and 2013, respectively. Medical tourism and the high influx of foreign workers were postulated to contribute to the increased number of TB cases seen in Singapore. ${ }^{(3)}$ However, this was not reflected in the present study, where the percentage of non-Singaporean residents and non-resident cases notified remained stable over the five years $(p=0.783)$.

We did not find any racial predilection for TB infection. While the racial distribution of TB cases notified at SGH was proportional to Singapore's racial distribution, ${ }^{(4)}$ the racial distribution of TB cases in Singapore (as reported by the Ministry of Health, Singapore) showed that Malays had the highest incidence of TB. ${ }^{(2)}$ Our study found that male Singaporean residents accounted for more than twice the number of TB cases as compared to female Singaporean residents; this concurs with the ministry's data, which stated that male patients made up $64.0 \%$ of new TB cases notified in Singapore. ${ }^{(2)}$ However, this gender disparity was not seen among the non-Singaporean residents and non-residents - the higher representation of women in this group could be due to the large number of foreign domestic workers. The higher percentage of TB cases notified in Singapore among non-Singaporean residents and non-residents within the 20-29 years age group is also likely to be related to the foreign worker population. Additional stringent screenings should perhaps be mandated as part of their pre-employment screening.

In Singapore, $9.0 \%$ of the population comprises elderly persons (i.e. aged $>65$ years). ${ }^{(4)}$ In the present study, we found that most $(31.9 \%)$ of the patients notified to have TB at SGH were elderly. This is similar to the percentages reported for Singapore ${ }^{(2)}$ and other developed countries; the elderly accounted for $36.9 \%$ of the annual TB notifications in Hong Kong ${ }^{(5)}$ and $20 \%$ in the United States. ${ }^{(6)}$ The higher risk of TB among the elderly was ascribed to various factors, including prior infection early in life (the geriatric population represents the largest reservoir of TB infection, particularly in developed nations), ${ }_{1}^{(7)}$ immunosenescence, malnutrition, poverty, decreased access to health services and coexisting medical diseases. ${ }^{(8,9)}$ The ageing population in Singapore is a phenomenon that will persist due to the country's decreasing birth rates and increasing life expectancy. Given that TB incidence rates are higher among the elderly, there is a need to step up efforts to manage this vulnerable population. This can be done by increasing vigilance, promoting awareness and having a high index of suspicion for unusual, nonspecific clinical and radiological presentations. ${ }^{(10-14)}$ These measures can facilitate early diagnosis and appropriate treatment, thereby minimising transmission of this infectious disease, and reducing its morbidity and mortality.

While extrapulmonary TB accounted for $14 \%$ of all TB cases in Singapore, ${ }^{(2)}$ we found a consistently higher percentage (27.1\%) of extrapulmonary TB among all the cases notified at SGH. This higher percentage could be explained by the hospital's patient case mix; $9.9 \%$ of the patients with extrapulmonary TB were immunocompromised, nearly half from HIV, which predisposes them to the development of extrapulmonary TB.

With respect to HIV testing in patients newly diagnosed with $\mathrm{TB}^{(15)}$ we found that there was better adherence to published guidelines, in that an upward trend in HIV testing, from $43.8 \%$ 
in 2006 to $64.2 \%$ in 2010 was observed (Fig. 4). This was a marked improvement from the previously published data for Singapore ${ }^{(16)}$ and comparable to the $68 \%$ reported for the United States in 2003. ${ }^{(17)}$ The percentage of HIV testing reported in 2010 (i.e. $64.2 \%$ ) was also higher than the $46 \%$ reported globally in 2012.(1) A HIV-positive person has increased susceptibility to TB (through the reactivation of latent infection or from a new TB infection), with rapid progression to active disease. Not knowing a patient's HIV status would compromise the patient's TB management and outcome. ${ }^{(18)}$ While the potential returns (in terms of reducing morbidity, mortality and sustained transmission) from HIV testing for TB patients are greatest in countries that have high coinfection rates, the resources necessary to maximise the clinical and preventive benefits of screening are more readily available in high-income countries. Therefore, we should strive to achieve $100 \%$ HIV testing in all patients newly diagnosed with TB in Singapore. If we assume that the patients who did not undergo HIV testing were seronegative, our HIV coinfection rate with TB would be 3.8\% (14/368) in 2006 and 2.9\% (12/407) in 2010; in other words, the coinfection rate with TB remained stable. However, the aforementioned coinfection rates are slightly higher than the $2.7 \%$ rate of HIV-positive TB patients reported for Singapore. ${ }^{(2)}$ This difference could be due to the patient case mix seen at our hospital.

In the present study, the overall mortality rate throughout the five years remained constant at $12.0 \%$. Death was mainly due to cancer, ischaemic heart disease and infections other than TB. Among the 28 (1.4\%) patients who died from TB, 75.0\% were smear-positive and $22(78.6 \%)$ patients were diagnosed on their first presentation to the hospital, suggesting that medical attention was sought very late in the course of illness. Increasing public awareness of the signs and symptoms of TB and encouraging early medical consultation may improve TB mortality rates. The remaining six patients had delayed TB diagnosis due to confounding medical illnesses. Thus, as we endeavour to minimise mortality, there is also a need to heighten our index of suspicion of TB, so that expedient diagnosis and treatment can be delivered.

To conclude, TB continues to result in significant morbidity and mortality in Singapore, and thus, there is no room for complacency in our fight against this infectious disease. Efforts should be concentrated on the elderly population, as this vulnerable group is at a high risk of developing TB.

\section{REFERENCES}

1. World Health Organization. Global tuberculosis report 2013. Available at: http://www.who.int/tb/publications/global_report/en/. Accessed April 4, 2014.

2. Ministry of Health, Singapore. Communicable Diseases Surveillance in Singapore 2012. Available at: http://www.moh.gov.sg/content/moh_web/ home/Publications/Reports/2013/Communicable_Diseases_Surveillance_ in_Singapore_2012.html. Accessed July 21, 2014.

3. Cutter J, Wang YT. Tuberculosis - an under-appreciated disease. Ann Acad Med Singapore 2010; 39:261-2.

4. Ministry of Health, Singapore. Population and Vital Statistics. Available at: https://www.moh.gov.sg/content/moh_web/home/statistics/Health_Facts_ Singapore/Population_And_Vital_Statistics.html. Accessed April 4, 2014.

5. Leung CC, Yew WW, Chan CK, et al. Tuberculosis in older people: a retrospective and comparative study from Hong Kong. J Am Geriatr Soc 2002; 50:1219-26.

6. Centers for Disease Control and Prevention (CDC). Trends in tuberculosis United States, 2005. MMWR Morb Mortal Wkly Rep 2006; 55:305-8.

7. Davies PD. Tuberculosis in the elderly: an international perspective. Clin Geriatr 1997; 5:15-26.

8. Aw D, Silva AB, Palmer DB. Immunosenescence: emerging challenges for an ageing population. Immunology 2007; 120:435-46.

9. Cruz-Hervert LP, Garcia-Garcia L, Ferreyra-Reyes L, et al. Tuberculosis in ageing: high rates, complex diagnosis and poor clinical outcomes. Age Ageing 2012; 41:488-95.

10. Couser JI Jr, Glassroth J. Tuberculosis. An epidemic in older adults. Clin Chest Med 1993; 14:491-9.

11. Pérez-Guzmán C, Vargas MH, Torres-Cruz A, Villarreal-Velarde H. Does aging modify pulmonary tuberculosis: A meta-analytical review. Chest 1999; 116:961-7.

12. Rajagopalan S, Yoshikawa TT. Tuberculosis in the elderly. Z Gerontol Geriat 2000; 33:374-80.

13. Packham S. Tuberculosis in the elderly. Gerontology 2001; 47:175-9.

14. Rajagopalan S. Tuberculosis and aging: a global health problem. Clin Infect Dis 2001; 33:1034-9.

15. Blumberg HM, Burman WJ, Chaisson RE, et al; American Thoracic Society, Centers for Disease Control and Prevention and the Infectious Diseases Society. American Thoracic Society/Centers for Disease Control and Prevention/Infectious Disease Society of America: treatment of tuberculosis. Am J Respir Crit Care Med 2003; 167:603-62.

16. Low SY, Eng P. Human immunodeficiency virus testing in patients with newly-diagnosed tuberculosis in Singapore. Singapore Med J 2009; 50:479-81.

17. Centers for Disease Control and Prevention (CDC). Reported HIV status of tuberculosis patients - United States, 1993-2005. MMWR Morb Mortal Wkly Rep 2007; 56:1103-6.

18. World Health Organization. WHO policy on collaborative TB/HIV activities: guidelines for national programmes and other stakeholders. Geneva, 2012. Available at: http://www.who.int/tb/publications/2012/ tb_hiv_policy_9789241503006/en. Accessed July 21, 2014. 